Portland State University

PDXScholar

$1-1-1994$

\title{
Numerical Simulation of Nonequilibrium Effects in an Argon Plasma Jet
}

C. H. Chang

Idaho National Engineering Laboratory

John D. Ramshaw

Portland State University, jdramshaw@yahoo.com

Follow this and additional works at: https://pdxscholar.library.pdx.edu/phy_fac

Part of the Physics Commons

Let us know how access to this document benefits you.

\section{Citation Details}

C.H. Change and J.D. Ramshaw, "Numerical simulation of nonequilibrium effects in an argon plasma jet," Phys. Plasmas 1, 3698 (1994)

This Article is brought to you for free and open access. It has been accepted for inclusion in Physics Faculty Publications and Presentations by an authorized administrator of PDXScholar. Please contact us if we can make this document more accessible: pdxscholar@pdx.edu. 


\title{
Numerical simulation of nonequilibrium effects in an argon plasma jet
}

\author{
C. H. Chang and J. D. Ramshaw \\ Idaho National Engineering Laboratory, Idaho Falls, Idaho 83415
}

(Received 14 January 1994; accepted 12 July 1994)

\begin{abstract}
Departures from thermal (translational), ionization, and excitation equilibrium in an axisymmetric argon plasma jet have been studied by two-dimensional numerical simulations. Electrons, ions, and excited and ground states of neutral atoms are represented as separate chemical species in the mixture. Transitions between excited states, as well as ionization/recombination reactions due to both collisional and radiative processes, are treated as separate chemical reactions. Resonance radiation transport is represented using Holstein escape factors to simulate both the optically thin and optically thick limits. The optically thin calculation showed significant underpopulation of excited species in the upstream part of the jet core, whereas in the optically thick calculation this region remains close to local thermodynamic equilibrium, consistent with previous experimental observations. Resonance radiation absorption is therefore an important effect. The optically thick calculation results also show overpopulations (relative to equilibrium) of excited species and electron densities in the fringes and downstream part of the jet core. In these regions, however, the electrons and ions are essentially in partial local thermodynamic equilibrium with the excited state at the electron temperature, even though the ionized and excited states are no longer in equilibrium with the ground state. Departures from partial local thermodynamic equilibrium are observed in the outer fringes and far downstream part of the jet. These results are interpreted in terms of the local relative time scales for the various physical and chemical processes occurring in the plasma.
\end{abstract}

\section{INTRODUCTION}

The assumption of local thermodynamic equilibrium (LTE) in thermal plasmas has been widely used both in simulations and in the interpretation of experimental data. In simulations, the LTE assumption significantly simplifies both the model and the computer code, thereby reducing the required developmental and computational effort. Experimentally, the interpretation of enthalpy probe measurements relies directly on the LTE assumption, since such measurements only provide information on the total enthalpy in the plasma, and not on how it is partitioned among the constituents. Similarly, emission spectroscopy measurements of the populations of upper excited states require the assumption of either complete or partial LTE to be converted into temperatures.

Departures from LTE in plasmas are of various different types. Translational or thermal nonequilibrium, ${ }^{1,2}$ in which the electrons have a different temperature from the heavy particles, occurs when the energy exchange rate between electrons and heavy particles is comparable to or slower than the time scales for plasma transport, provided a mechanism is present (e.g., an electric field and/or chemical reactions involving electrons) for preferentially heating or cooling the electrons or heavy particles. For example, thermal nonequilibrium is often associated with ionization nonequilibrium, since three-body recombination with electrons as the third body heats up the electrons via the chemical (ionization) energy release. ${ }^{3,4}$ Thermal nonequilibrium has been studied extensively in various plasma flows including electric arcs, ${ }^{5}$ radio-frequency (RF) plasmas, ${ }^{6}$ and plasma-solid wall interactions. $^{\text {? }}$

Ionization nonequilibrium commonly occurs at high plasma flow rates, where the fluid dynamical time scales which control plasma cooling are comparable to the relaxation time for energy equilibrium and/or recombination of electrons and ions. In particular, strong departures from ionization equilibrium are observed in low pressure plasma jets, ${ }^{3,4}$ where the flow is usually supersonic and collision frequencies for chemical reactions are low. Ionization nonequilibrium has also been studied in electric ares ${ }^{5}$ and in shock waves associated with reentry problems. ${ }^{8,9}$ Thermal and/or ionization nonequilibrium are very important in thermal plasma processing, since they have strong effects on both the macroscopic plasma flow ${ }^{3-5}$ and the heating of entrained particles. ${ }^{7}$

Nonequilibrium effects can also occur in regions of steep gradients of temperatures or species concentrations, such as the fringes of plasma jets or electric ares, where time scales for energy or species transport can become comparable to chemical or thermal relaxation time scales. Departures from both thermal and ionization equilibrium have been reported $^{3-5}$ in the fringes of such plasmas even in situations where LTE prevails in the plasma core. Similar nonequilibrium effects occur in the boundary layer around a particle. ${ }^{7}$

Analogous considerations apply to the populations of excited electronic states of atoms and ions in the plasma. Departures from excitational equilibrium may be expected to occur when the time scales for collisional and radiative transitions between excited states are comparable to those for convection and diffusion in the plasma. Such departures have recently been studied using an idealized zero-dimensional collisional-radiative (CR) model for excited state kinetics. ${ }^{10.11}$ A primary objective of the present work is to extend such studies to include interactions with convective and diffusive effects. Analogous departures from vibrational equilibrium in recombining diatomic gases were observed some time ago. ${ }^{12}$ 
The populations of upper excited states are important in the interpretation of emission spectroscopic measurements. According to the results of CR model calculations by Repetti et al. ${ }^{11}$ and measurements by Owano et al. ${ }^{13}$ regardless of whether lower excited states are in equilibrium or not, upper excited states were found to be in partial local thermodynamic equilibrium (PLTE) with electrons when the electron density was higher than about $10^{15} \mathrm{~cm}^{-3}$. Gordon and Kruger $^{14}$ observed that small amounts of hydrogen selectively quench the first excited state of argon, thereby driving argon plasmas away from excitation equilibrium. This departure from PLTE reflects the disparity between the time scales for quenching and transitions between excited states. Cho and Eddy ${ }^{15}$ also observed departures from PLTE in hydrogen plasmas, which they interpreted in terms of a more complex generalized multithermal equilibrium (GMTE) model.

In this paper, departures from thermal, chemical, and excitation equilibrium in an argon plasma jet are studied by direct two-dimensional simulations using a two-temperature multicomponent fluid dynamical model with chemical and excited state kinetics. These simulations are not intended to correspond to any particular experiment, but the conditions are generally typical of plasma jets used in plasma processing. ${ }^{16,17}$ For comparison purposes, we also performed a similar simulation with much lower values of the torch power and argon flow rate. To our knowledge, this study represents the first complete numerical simulations of multidimensional plasma flow with excited state kinetics. Previous studies of excited state kinetics have taken a more empirical approach in which experimental data is used in conjunction with zero-dimensional CR models, ${ }^{14,15}$ while previous one-dimensional ${ }^{5,9}$ and multidimensional ${ }^{3,4,7,13}$ nonequilibrium plasma flow studies have neglected excited state kinetics. The present simulations are fully self-consistent in all respects, so that all effects due to time scale disparities are fully and automatically accounted for. Indeed, a leitmotiv of the present work is that the essential physics of nonequilibrium behavior in thermal plasmas largely resides in the relative time scales of the various competing physical and chemical processes, including fluid dynamical effects such as convection and diffusion as well as chemical and excitational rate processes. We elaborate on this theme by a rather detailed examination of the local time scales occurring in the plasma.

We represent electrons, ions, and atomic ground and excited states as separate chemical species in the plasma mixture. All important rate processes are treated as separate kinetic chemical reactions, including ionization/recombination reactions and collisional and radiative transitions between ground and excited states. Reaction rates are obtained from the collisional-radiative (CR) model described by Braun and Kunc. ${ }^{10}$ Thermal nonequilibrium is represented by a conventional two-temperature model. ${ }^{1,2}$ Diffusion of charged species in plasmas is controlled by the local electric field which spontaneously develops to preserve zero current density and local charge neutrality. Diffusion under these conditions is referred to as ambipolar diffusion,' suitable formulations for which have recently been developed for arbitrary multicom- ponent plasmas. ${ }^{18,19}$ The present simulations include the twotemperature version of this formulation. ${ }^{19}$

To examine excitation equilibrium in plasmas, it is necessary to allow for the transport of resonance radiation associated with transitions between the ground and first excited state. For this purpose it would be desirable to include a detailed quantitative description of radiative transport, allowing for the large variations in photon mean free path with wavelength. However, this would be a much more ambitious undertaking. We have therefore adopted a simplified approach in order to capture the qualitative effects of resonance radiation transport on excitation nonequilibrium. This approach uses Holstein escape factors ${ }^{20,21}$ to represent resonance radiation transport in terms of the "net" radiation escaping from the plasma. Even though Holstein escape factors have only been derived for simple geometries ${ }^{20,21}$ (such as plane and cylindrical), they may still be used to represent the two extreme cases of optically thick and thin plasmas, corresponding to complete self-absorption and escape of resonance radiation, respectively.

The entire system of governing equations described above constitutes a comprehensive computational model for nonequilibrium thermal plasmas. This model is embodied in the LAVA computer code, the single-temperature version of which has previously been described. ${ }^{16}$ The original version of LAVA has now been extended to two temperatures, and the resulting current version is capable of handling either singleor two-temperature multicomponent plasma flows with arbitrary numbers of species and reactions.

The present calculations have been performed for the two extreme situations of optically thick and thin plasmas in order to obtain insight into the importance of the various effects. This illustrates the capability of modeling to isolate individual processes and assess their effects in ways that are often difficult or impossible to accomplish experimentally. Departures from ionization, excitation, and thermal equilibrium are all observed in different regions of the flow field, and these departures are correlated with the relative time scales occurring in the plasma. Departures from excitation equilibrium occur in most of the flow field, due to the fact that the time scale for deexcitation is relatively slow and forms a "bottleneck" or rate-limiting process. In the physically more realistic optically thick case, we find that the electrons and ions in the plasma core are essentially in PLTE with the excited state at the electron temperature, even though the ionized and excited states are not in equilibrium with the ground state. This is consistent with previous observations ${ }^{11,13,14}$ of PLTE of excited states, and also correlates well with the regions where the time scale for recombination is much shorter than the other time scales. In contrast, we find significant departures from PLTE in the fringes of the jet, and to a lesser degree in the downstream region, and this can again be understood in terms of the relative time scales. To our knowledge, the present investigation is the first theoretical study of PLTE based on a complete simulation in which all of the various physical effects and associated time scales are simultaneously and self-consistently included. 


\section{FLUID DYNAMICAL MODEL}

The fluid dynamical equations solved by LAVA consist of momentum and thermal internal energy equations for the multicomponent fluid mixture, continuity equations for each component of the mixture, and state and constitutive relations. Viscous stresses and thermal conduction are included in full generality, while species diffusion is represented by a self-consistent effective binary diffusion approximation, ${ }^{22.23}$ suitably generalized to allow for ambipolar diffusion of charged species in both thermal equilibrium ${ }^{18}$ and nonequilibrium. ${ }^{19} \mathrm{~A}$ detailed description of the governing equations for LTE plasmas is given elsewhere. ${ }^{16}$ The present discussion will therefore concentrate on the extension of the model to nonequilibrium.

The species conservation equations are given by

$$
\begin{aligned}
& \frac{\partial \rho_{i}}{\partial t}+\nabla \cdot\left(\rho_{i} \mathbf{u}\right)=-\nabla \cdot \mathbf{J}_{i}+\dot{\rho}_{i}^{c}, \\
& \frac{\partial \rho}{\partial t}+\nabla \cdot(\rho \mathbf{u})=0,
\end{aligned}
$$

where $\rho_{i}$ is the partial mass density (mass per unit volume) of species $i, \mathbf{u}$ is the fluid velocity, $\mathbf{J}_{i}$ is the diffusional mass flux of species $i, \dot{\rho}_{i}^{c}$ is the rate of change of $\rho_{i}$ due to chemical reactions, ${ }^{16}$ and $\rho=\Sigma_{i} \rho_{i}$ is the total mass density of the fluid. The diffusion fluxes $\mathbf{J}_{i}$ are determined by a selfconsistent effective binary diffusion approximation, ${ }^{22.23}$ generalized to allow for ambipolar diffusion of charged particles in two-temperature plasmas. ${ }^{19}$ Under the assumption that pressure and thermal diffusion are negligible compared to ordinary (concentration) diffusion, the resulting expression for $\mathbf{J}_{i}$ for all species except electrons is given by

$$
\begin{aligned}
\mathbf{J}_{i}= & -\frac{p M_{i}\left(D_{i}+D_{t}\right)}{R_{g} T} \nabla\left(\frac{p_{i}}{p}\right) \\
& +\frac{\rho_{i}}{\rho} \sum_{j \neq e} \frac{p M_{j}\left(D_{j}+D_{t}\right)}{R_{g} T} \nabla\left(\frac{p_{j}}{p}\right)+\mathbf{A}_{i},
\end{aligned}
$$

where subscript $e$ denotes the free electrons, $M_{i}$ is the molecular weight of species $i, D_{i}$ is the laminar effective binary diffusivity of species $i,{ }^{22,23} D_{t}=\mu_{t} / \rho \mathrm{Sc}_{t}$ is the turbulent diffusivity, $\mu_{t}$ is the turbulent viscosity (defined later), $S c_{t}$ is the turbulent Schmidt number, $p_{i}$ is the partial pressure of species $i, p=\Sigma_{i} p_{i}$ is the total pressure, $T$ is the heavy particle temperature, $R_{g}$ is the universal gas constant, and $\mathbf{A}_{i}$ is the ambipolar forced diffusion flux given by

$$
\begin{aligned}
\mathbf{A}_{i}= & \frac{1}{R_{g} T}\left(M_{i} q_{i} \rho_{i}\left(D_{i}+D_{t}\right)-\frac{\rho_{i}}{\rho} \sum_{j \neq e} M_{j} q_{j} \rho_{j}\left(D_{j}\right.\right. \\
& \left.\left.+D_{i}\right)\right) \frac{\nabla p_{e}}{q_{e} \rho_{e}}
\end{aligned}
$$

where $q_{i}$ is the electric charge per unit mass of species $i$. The diffusional flux of electrons is then given by

$$
\mathbf{J}_{e}=-\frac{1}{q_{e}} \sum_{j \neq e} q_{j} \mathbf{J}_{j}
$$

The momentum equation can be written as

$$
\frac{\partial(\rho \mathbf{u})}{\partial t}+\nabla \cdot(\rho \mathbf{u u})=-\nabla\left(p+\frac{2}{3} \rho k\right)+\nabla \cdot \boldsymbol{\sigma}
$$

where $k$ is the turbulent kinetic energy per unit mass. The viscous stress tensor $\boldsymbol{\sigma}$ is given by

$$
\boldsymbol{\sigma}=\left(\mu+\mu_{t}\right)\left[\boldsymbol{\nabla} \mathbf{u}+(\boldsymbol{\nabla} \mathbf{u})^{T}\right]+\left(\lambda+\lambda_{t}\right)(\boldsymbol{\nabla} \cdot \mathbf{u}) \mathbf{I},
$$

where $\mu$ is the molecular viscosity, $\lambda$ is the second viscosity coefficient, ${ }^{24} \lambda_{t}=-(2 / 3) \mu_{t}, I$ is the unit dyadic, and superscript $T$ denotes the transpose. The laminar bulk viscosity is $\lambda+(2 / 3) \mu$, which vanishes for monatomic gases.

Thermal nonequilibrium is represented by solving separate energy equations for the total thermal internal energy of the mixture and for the thermal internal energy of the free electrons. These equations are given by ${ }^{1.2}$

$\frac{\partial(\rho e)}{\partial t}+\nabla \cdot(\rho e \mathbf{u})=-p \nabla \cdot \mathbf{u}-\nabla \cdot \mathbf{q}+\rho \epsilon+\dot{Q}^{c}$,

$\frac{\partial\left(\rho_{e} e_{e}\right)}{\partial t}+\nabla \cdot\left(\rho_{e} e_{e} \mathbf{u}\right)=-p_{e} \boldsymbol{\nabla} \cdot \mathbf{u}-\nabla \cdot \mathbf{q}_{e}+\dot{\rho}_{e}^{c} e_{e}+\dot{Q}_{e}^{c}+\dot{Q}_{e h}$,

where $\epsilon$ is the viscous dissipation rate, $e$ is the total thermal internal energy per unit mass, $\dot{Q}^{c}$ is the rate of change of $\rho e$ due to chemical reactions, ${ }^{16} e_{e}$ and $\dot{Q}_{e}^{c}$ are the corresponding quantities for the free electrons, and $\dot{Q}_{e h}$ is the energy exchange rate between electrons and heavy particles (defined later). Energy losses due to the escape of optically thin radiation from the plasma are modeled by omitting the corresponding terms from $\dot{Q}^{c}$ and $\dot{Q}_{e}^{c}$, so that the radiation energy is lost to the system rather than being deposited in the plasma. This is why radiation loss terms do not explicitly appear in Eqs. (8) and (9). The radiative transitions to be treated in this way are described in Sec. III below.

The heavy particle energy is obtained by subtracting the electron energy from the total energy. The heat flux vectors $q$ and $\mathbf{q}_{e}$ contain contributions from both pure heat conduction and species diffusion, ${ }^{16}$ and take the form

$$
\begin{aligned}
& \mathbf{q}=-\left(K_{h}+\frac{c_{p h} \mu_{t}}{\operatorname{Pr}_{t}}\right) \nabla T-\left(K_{e}+\frac{c_{p e} \mu_{t}}{\operatorname{Pr}_{t}}\right) \nabla T_{e}+\sum_{i} h_{i} \mathbf{J}_{i} \\
& \mathbf{q}_{e}=-\left(K_{e}+\frac{c_{p e} \mu_{t}}{\operatorname{Pr}_{t}}\right) \nabla T_{e}+h_{e} \mathbf{J}_{e}
\end{aligned}
$$

where $K_{\hbar}$ and $K_{e}$ are the laminar thermal conductivities of heavy particles and electrons, respectively, $T_{e}$ is the electron temperature, $c_{p h}$ and $c_{p e}$ are, respectively, the heavy particle mixture and electron specific heats at constant pressure (defined later), $\mathrm{Pr}_{t}$ is the turbulent Prandtl number, and $h_{i}$ is the partial specific enthalpy of species $i$. In laminar flow $p \epsilon=\Phi$ $=\sigma: \nabla u$, while $\epsilon$ in turbulent flow is obtained by solving a transport equation as described below.

The thermodynamic state relations must be generalized to allow for two temperatures. Thus the total thermodynamic pressure is given by 


$$
p=p_{h}+p_{e}=\sum_{i \neq e} \frac{\rho_{i}}{M_{i}} R_{g} T+\frac{\rho_{e}}{M_{e}} R_{g} T_{e} .
$$

The total thermal internal energy is related to the temperatures by

$$
\rho e=\sum_{i \neq e} \rho_{i} e_{i}(T)+\rho_{e} e_{e}\left(T_{e}\right),
$$

where $e_{i}(T)$ is the partial specific thermal internal energy of species $i$ at temperature $T$. The specific heats at constant volume and pressure are given by

$$
\begin{aligned}
& \rho_{h} c_{v h}=\sum_{i \neq e} \rho_{i} \frac{d e_{i}}{d T}, \\
& c_{v e}=\frac{d e_{e}}{d T_{e}}, \\
& \rho_{h} c_{p h}=\sum_{i \neq e} \rho_{i}\left(\frac{d e_{i}}{d T}+\frac{R_{g}}{M_{i}}\right), \\
& c_{p e}=\frac{d e_{e}}{d T_{e}}+\frac{R_{g}}{M_{e}},
\end{aligned}
$$

where $\rho_{h}=\Sigma_{i \neq e} \rho_{i}$. Notice that the above energies and enthalpies are purely thermal and do not include heats of formation.

The energy exchange rate between electrons and heavy particles is given by ${ }^{9}$

$$
\dot{Q}_{e h}=3 \frac{m_{e}}{m_{h}} k_{\mathrm{B}} n_{e}\left(T-T_{e}\right)\left(\nu_{e i}+\nu_{e a}\right),
$$

where $n_{k}$ and $m_{k}$ are, respectively, the number density and mass of species $k$, and $k_{\mathrm{B}}$ is Boltzmann's constant. The collision frequency $\nu_{e i}$ between electrons and ions is obtained using the cutoff Coulomb potential,,${ }^{9,10}$ while the electronneutral collision frequency $\nu_{e a}$ is obtained from published collision cross-section data. ${ }^{9,10}$

In the present simulations, the $k-\epsilon$ turbulence model ${ }^{16}$ is used to represent the effects of turbulence. Transport equations are solved for the turbulent kinetic energy per unit mass $k$ and its dissipation rate $\epsilon$,

$$
\begin{aligned}
\frac{\partial(\rho k)}{\partial t} & +\nabla \cdot(\rho k \mathbf{u}) \\
= & -\frac{2}{3} \rho k \boldsymbol{\nabla} \cdot \mathbf{u}+\boldsymbol{\nabla} \cdot\left[\left(\mu+\mu_{t}\right) \nabla k\right]+\Phi-\rho \epsilon,
\end{aligned}
$$

$$
\begin{aligned}
\frac{\partial(\rho \epsilon)}{\partial t}+\nabla \cdot(\rho \epsilon \mathbf{u})= & \left(c_{3}-\frac{2}{3} c_{1}\right) \rho \epsilon \nabla \cdot \mathbf{u}+\nabla \cdot\left[\left(\mu+\frac{\mu_{t}}{\sigma_{\epsilon}}\right) \nabla \epsilon\right] \\
& +\frac{\epsilon}{k}\left(c_{1} \Phi-c_{2} \rho \epsilon\right),
\end{aligned}
$$

where the coefficients are given by $c_{1}=1.44, c_{2}=1.92$, $c_{3}=-1.0$, and $\sigma_{\epsilon}=1.3$. The turbulent viscosity $\mu_{t}$ is then given by

$$
\mu_{t}=\frac{c_{\mu} \rho k^{2}}{\epsilon}
$$

where $c_{\mu}=0.09$. This is the same version of the $k-\epsilon$ model described and used in previous studies, ${ }^{16,17}$ with the same values of the various parameters. In particular, we use the values $\mathrm{Pr}_{t}=\mathrm{Sc}_{t}=0.7$ instead of the more conventional values $\mathrm{Pr}_{t}=\mathrm{Sc}_{t}=0.9$. As is well known, $k-\epsilon$ models have particular difficulties in dealing with axisymmetric and swirling jets, for which various ad hoc correction terms ${ }^{25,26}$ have frequently been employed. The effects of these correction terms for plasma jets have previously been studied, ${ }^{17}$ and none produced significantly better results than the basic model with no correction terms. In the present simulations, such correction terms were therefore omitted.

Simple $k-\epsilon$ models of this type are not really satisfactory even for simple incompressible flows, for which they often require ad hoc corrections. It would therefore be most remarkable if such models were quantitatively applicable to thermal plasmas without further major modifications, so this should not be expected. However, such models usually do provide useful semiquantitative results of fair accuracy, ${ }^{17,27}$ and one may reasonably hope that this will continue to be the case in simulations of the present type.

\section{COLLISIONAL-RADIATIVE (CR) MODEL}

In the present model, electrons, ions, and excited and ground states of neutral atoms are treated as separate species in the mixture. All collisional and radiative transitions are treated as separate chemical reactions between these species. Reaction rates are then to be obtained from existing collisional-radiative (CR) models. In the present simulations, we adopted the three-level atomic model described by Braun and Kunc. ${ }^{10}$ Four lower excited states $\left[3 p^{5} 4 s^{1} P_{1}\right.$ and $\left.3 p^{5} 4 s\left({ }^{3} P_{0},{ }^{3} P_{1},{ }^{3} P_{2}\right)\right]$ separated by very small energy gaps are lumped together and treated as a single excited state $p$, while subscripts $c$ and $s$ refer to the ionized and ground states, respectively. All higher excited states are neglected. The following reactions (transitions) are included in the model

$$
\begin{aligned}
& \mathrm{Ar}_{s}+e^{-} \rightleftharpoons \mathrm{Ar}_{p}+e^{-}, \\
& \mathrm{Ar}_{s}+e^{-} \rightleftharpoons \mathrm{Ar}^{+}+e^{-}+e^{-}, \\
& \mathrm{Ar}_{p}+e^{-} \rightleftharpoons \mathrm{Ar}^{+}+e^{-}+e^{-}, \\
& \mathrm{Ar}_{p} \rightarrow \mathrm{Ar}_{s}+h \nu, \\
& \mathrm{Ar}^{+}+e^{-} \rightarrow \mathrm{Ar}_{s}+h \nu, \\
& \mathrm{Ar}^{+}+e^{-} \rightarrow \mathrm{Ar}_{p}+h \nu .
\end{aligned}
$$

The first three reactions represent collisional transitions and ionization/recombination processes by electron impact, and the remaining three represent the net radiative decay of excited and ionized species. Other collisional processes are neglected. Collisional rates for upward and downward transitions as well as the effective (net) radiative decay rates $A^{\text {eff }}$ are obtained as described by Braun and Kunc. ${ }^{10}$ The plasma is assumed to be optically thin to the continuum radiation due to recombination processes, so the effective rates $A_{c p}^{\text {eff }}$ and $A_{c s}^{\text {eff }}$ for radiative recombination are the same as the corresponding true rates $A_{c p}$ and $A_{c s} .{ }^{10}$ The effective rate $A_{p s}^{\text {eff }}$ 
for spontaneous emission of resonance radiation is obtained by multiplying the true rate $A_{p s}$ by the Holstein escape factor $\kappa_{p s}{ }^{20,21}$

The Holstein escape factor is a macroscopic parameter which represents the fraction of the resonance radiation leaving the plasma. The optically thick case $\left(\kappa_{p s}=0\right)$ corresponds to complete self-absorption of the $p-s$ spectral line, while the optically thin case $\left(\kappa_{p s}=1\right)$ corresponds to complete escape of the line radiation. The use of this escape factor replaces a detailed radiation transport calculation, which would be much more difficult and time consuming. Holstein escape factors have been systematically derived only for simple geometries, but they may be used more generally to represent resonance radiation transport in the two extreme cases of optically thick $\left(\kappa_{p s}=0\right)$ and optically thin $\left(\kappa_{p s}=1\right)$ plasmas, and this is what is done in the present study.

In the present simulations, the time scales associated with these transitions/reactions range over more than ten orders of magnitude due to the wide range of temperatures and species densities encountered in the flow field. This range of time scales includes characteristic times very short compared to those associated with fluid dynamical effects such as convection. The presence of these very short time scales precludes the use of conventional explicit or linearly implicit schemes for coupling these kinetic processes to the fluid flow. We therefore use a fully implicit algorithm for treating an arbitrary system of coupled fast and slow chemical reactions in fluid dynamics. ${ }^{28}$

\section{COMPUTATIONAL RESULTS}

The model described above has been used to simulate a turbulent argon plasma jet flowing into a cold argon environment. The calculational region is $6 \mathrm{~cm}$ radially by $12 \mathrm{~cm}$ axially, and is subdivided by a nonuniform $40 \times 68$ computational mesh as shown in Fig. 1. The torch geometry corresponds to a typical commercial plasma torch (Miller SG100 ). The inside radius of the torch nozzle at the exit is $R_{i}=4$ $\mathrm{mm}$. The outside radius of the torch housing is $R_{0}=33.3 \mathrm{~mm}$. The geometry is axisymmetric, so the simulations were performed in two-dimensional cylindrical coordinates. The radial coordinate is $r$ and the axial coordinate is $z$. The left boundary is the symmetry axis, the nozzle exit plane is at $z=0$, and the flow is upward. The torch body portion of the bottom boundary is treated as a solid wall. The remainder of the bottom boundary, as well as the top and right boundaries, are open boundaries at which the pressure is assumed to be ambient. The flow at these boundaries is calculated rather than imposed, so it can be either inward or outward at different locations on the boundary. Where inflow exists, the properties of the inflowing gas are taken to be those of the ambient cold argon.

Radial profiles of axial, radial, and swirl velocity, temperature, species densities, and turbulence parameters at the torch exit are required as inflow boundary conditions for the calculations. Frequently some or all of these profiles are not known, and it is then necessary to assume their forms subject to the constraints provided by the given values of argon mass flow rate $W$ and net torch power $P$. In the present calcula-

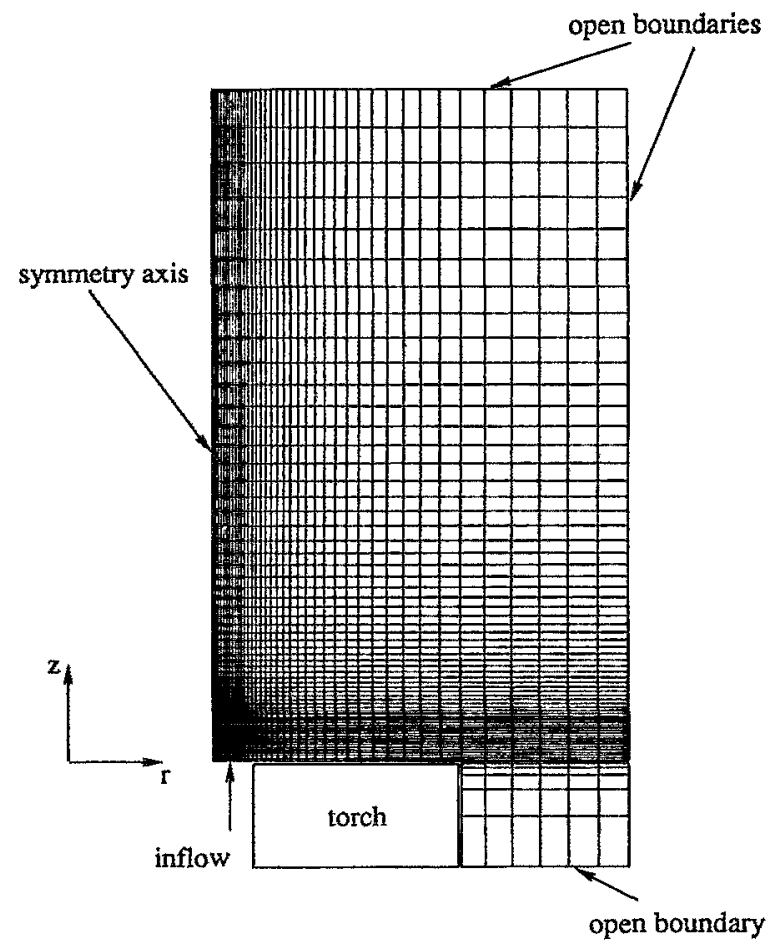

FIG. 1. Calculation domain and computational mesh.

tions the swirl velocity was taken to be zero, ${ }^{17}$ and the axial velocity and temperature profiles at the torch exit plane $z=0$ were taken to be of the form

$$
\begin{aligned}
& v=v_{0}\left[1-\left(\frac{r}{R_{i}}\right)^{n}\right], \\
& T=\left(T_{0}-T_{w}\right)\left[1-\left(\frac{r}{R_{i}}\right)^{m}\right]+T_{w},
\end{aligned}
$$

where $T_{\kappa}=700 \mathrm{~K}$, and the parameters $v_{0}, T_{0}, n$, and $m$ are related to $W$ and $P$ via the relations ${ }^{16}$

$$
\begin{aligned}
W= & \rho_{\mathrm{amb}} \dot{V}=2 \pi \int_{0}^{R_{i}} \rho v r d r \\
P= & 2 \pi \sum_{i} \int_{0}^{R_{i}} \rho_{i}\left(h_{i}(T)+\frac{\Delta H_{f_{i}}^{0}}{M_{i}}\right) v r d r-W\left(h_{\mathrm{Ar}_{\mathrm{s}}}\left(T_{\mathrm{amb}}\right)\right. \\
& \left.+\frac{\Delta H_{f \mathrm{Ar}_{s}}^{0}}{M_{\mathrm{Ar}}}\right)
\end{aligned}
$$

where $v$ is the axial velocity, $\rho_{\text {amb }}$ and $T_{\text {amb }}$ are, respectively, the density and temperature of the cold argon entering the torch, $\Delta H_{f i}^{0}$ is the heat of formation of species $i$ at absolute zero, and the $i$ summation runs over $\mathrm{Ar}_{s}, \mathrm{Ar}_{p}, \mathrm{Ar}^{+}$, and $e^{-}$. The parameter values used in the present simulations were $v_{0}=918.4 \mathrm{~m} / \mathrm{s}, T_{0}=12674 \mathrm{~K}, n=3$, and $m=9$, resulting in values of $\dot{V}=35.4 / / \mathrm{m}$, and $P=12.1 \mathrm{~kW}$, which are typical operating conditions for this torch. ${ }^{17}$ The species densities $\rho_{i}$ are obtained from $p$ and $T$ by assuming complete LTE at the inflow, including ionization, excitation, and thermal equilib- 


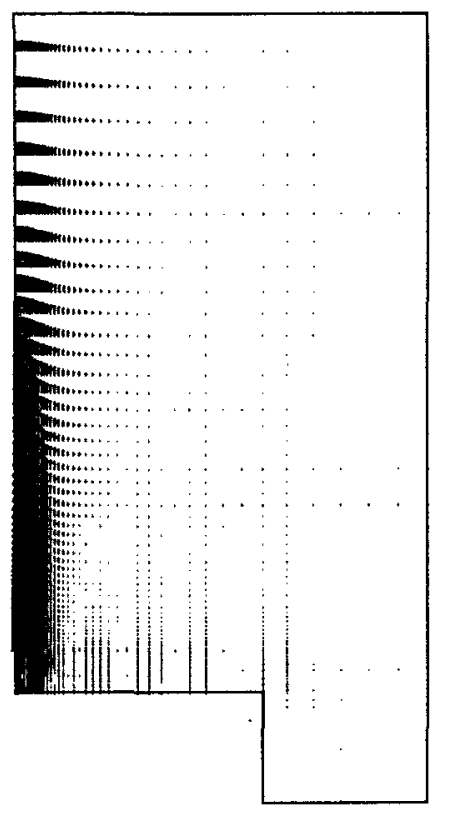

FIG. 2. Velocity vectors for optically thick case.

rium $\left(T_{e}=T\right)$. As will be seen, the present simulations predict significant departures from equilibrium in plasma jets at atmospheric pressure in spite of the fact that LTE is assumed at the inflow. In reality, it is likely that significant departures from equilibrium exist at the inflow as well, thereby further increasing the overall importance of nonequilibrium effects.

Information regarding inflow profiles of turbulence in plasma torches is not available. We simply assumed an inflow turbulent kinetic energy profile of the form ${ }^{27}$

$$
k(r)=k_{\max }\left|\frac{\partial v}{\partial r} /\left(\frac{\partial v}{\partial r}\right)_{\max }\right|,
$$

where $(\partial v / \partial r)_{\max }$ is the largest axial velocity gradient with respect to radial direction at the nozzle exit, and $k_{\max }$ is defined as

$$
k_{\max }=\frac{3}{2}\left(0.1 v_{0}\right)^{2} .
$$

The inflow profile of $\epsilon$ was then obtained from the turbulent kinetic energy profile by the prescription of Leschziner and Rodi. ${ }^{26}$

Calculations were performed for both $\kappa_{p s}=0$ (optically thick) and $\kappa_{p s}=1$ (optically thin). (The optically thin calculation was performed using a reduced $4 \mathrm{~cm} \times 10 \mathrm{~cm}$ computational region with a $35 \times 62$ mesh to save computer time without significantly affecting the results.) The calculations were initialized by filling the computational domain with quiescent ambient cold argon, which is then displaced by the incoming plasma until a statistically steady state is reached. (The flow field continues to fluctuate slightly, but the amplitude of the fluctuations is so small that they are visually indistinguishable.) Figure 2 shows velocity vectors for the optically thick case, and Fig. 3 shows velocity vectors with correct directions but logarithmically scaled lengths so that the entrainment of ambient argon can be seen. Figure 4

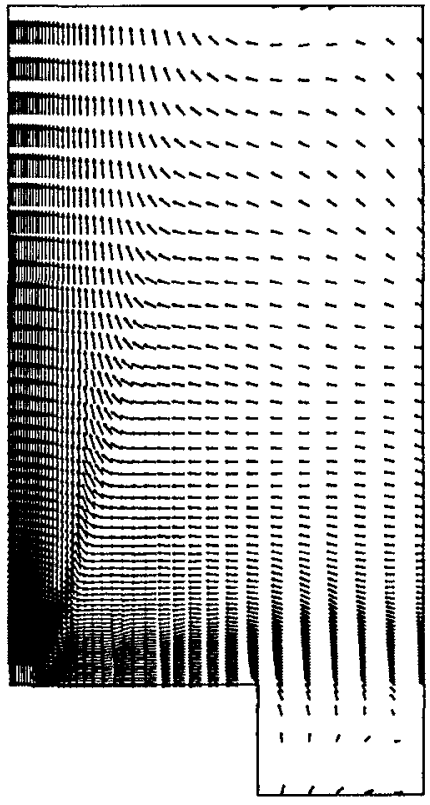

FIG. 3. Log-scale velocity vectors for optically thick case.

shows the corresponding heavy particle temperature field. The electron temperature field is visually indistinguishable from Fig. 4, indicating that the plasma is essentially in thermal equilibrium. The velocity vectors and temperature fields for the optically thin case are very similar.

Heavy particle and electron temperatures along the centerline of the jet in the two cases are shown in Figs. 5 and 6. As expected, thermal equilibrium prevails in the jet core for the optically thick case shown in Fig. 5. In the optically thin

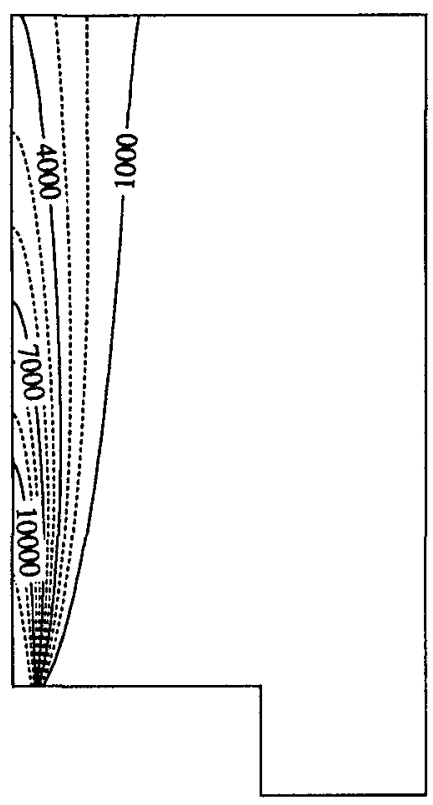

FIG. 4. Heavy particle temperature field for optically thick case. 


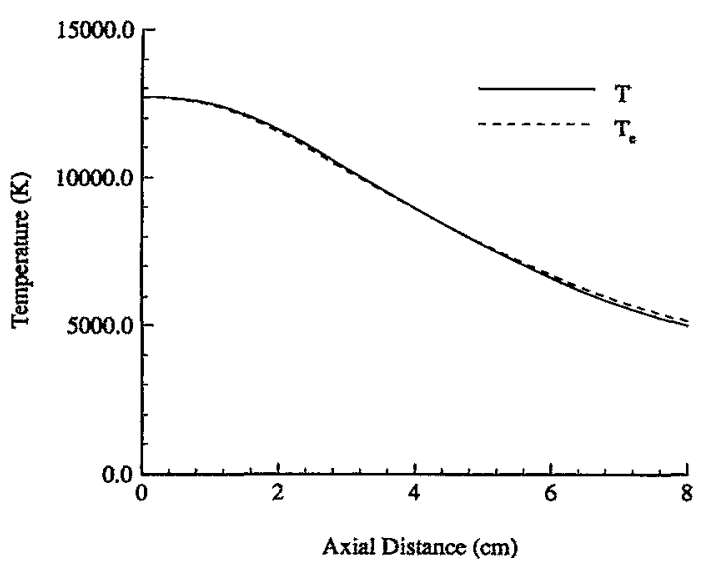

FIG. 5. Heavy particle and electron temperatures along the centerline for optically thick case.

case shown in Fig. 6, the electron temperature is seen to be lower than the heavy particle temperature in some parts of the plasma. In this case, resonance radiation escapes from the plasma, thereby enhancing radiative decay of the excited state and reducing its population. This in turn enhances collisional excitation by electrons, the energy for which is supplied by the electrons, thereby reducing their temperature.

Figures 7 and 8 show the degree of nonequilibrium for each reaction and the electron number density along the centerline. The degrees of nonequilibrium are defined as

$$
\begin{aligned}
& \zeta_{p s}=\frac{\left[\mathrm{Ar}_{p}\right]}{\left[\mathrm{Ar}_{s}\right] K_{p s}}, \\
& \zeta_{c s}=\frac{\left[\mathrm{Ar}^{+}\right]\left[e^{-}\right]}{\left[\mathrm{Ar}_{s}\right] K_{c s}}, \\
& \zeta_{c p}=\frac{\left[\mathrm{Ar}^{+}\right]\left[e^{-}\right]}{\left[\mathrm{Ar}_{p}\right] K_{c p}},
\end{aligned}
$$

where $[X]$ is the molar concentration of species $X$ in the plasma, and $K_{p s}, K_{c s}$, and $K_{c p}$, respectively, represent the

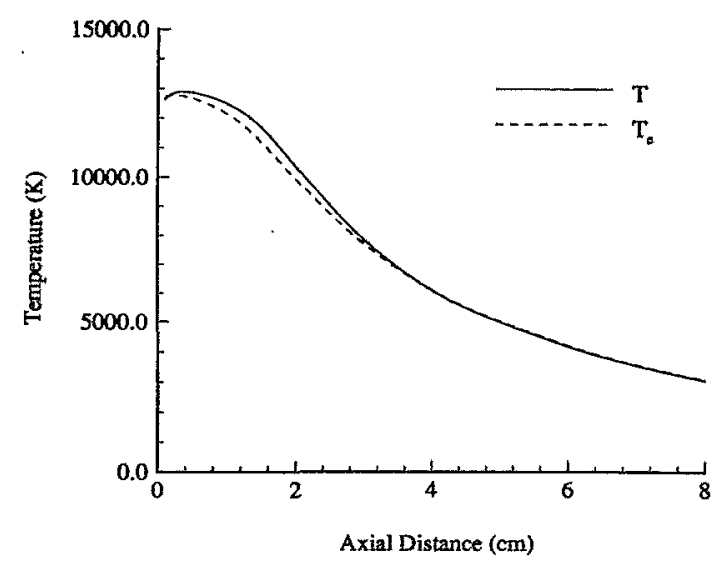

FIG. 6. Heavy particle and electron temperatures along the centerline for optically thin case.

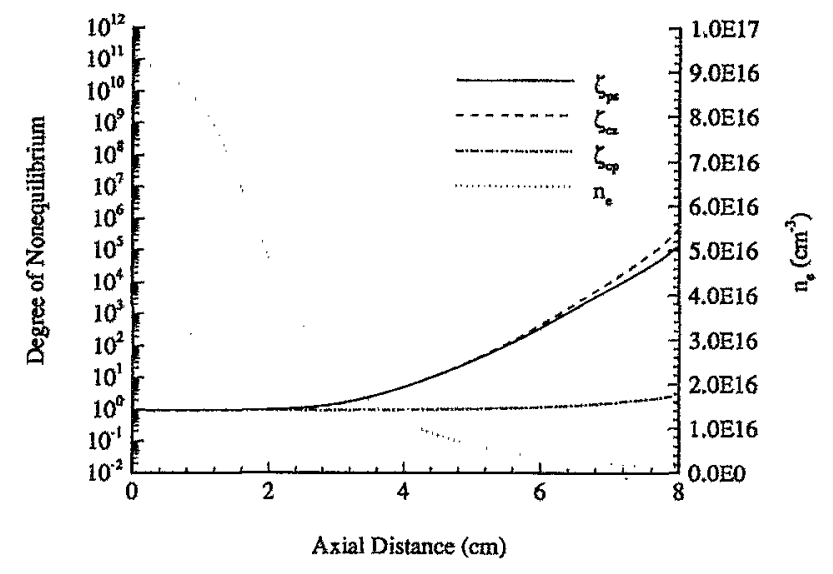

FIG. 7. Degree of nonequilibrium and electron density along the centerline for optically thick case.

equilibrium constants for excitation, and for ionization from the ground and excited states, evaluated at the electron temperature. Values $\zeta>1$ represent overpopulation of products or underpopulation of reactants, while $\zeta<1$ represents the opposite. As temperature and electron density decrease, ionization and excitation depart from equilibrium as expected for both cases, resulting in overpopulation of excited and ionized species. In the optically thick case shown in Fig. 7, ionization from the excited state stays close to equilibrium, confirming previous observations $s^{11,13}$ of PLTE of excited states with electrons at the electron temperature. In the optically thin case shown in Fig. 8, underpopulation of excited and ionized species relative to the ground state is observed for $0<z \leqslant 2.5 \mathrm{~cm}$, and this reduces the electron temperature as previously discussed and illustrated in Fig. 6.

Previous theoretical and experimental studies neglecting excited state kinetics ${ }^{3-5}$ have found that the core region of argon plasmas at atmospheric pressure is generally in ionization equilibrium. This is consistent with the present optically thick results in the region $0<z \leqslant 3 \mathrm{~cm}$, as shown in Fig. 7 .

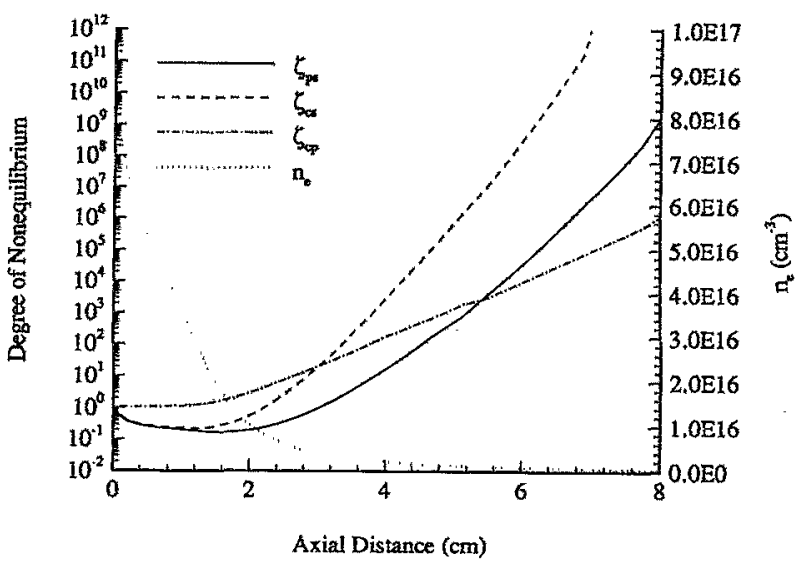

FIG. 8. Degree of nonequilibrium and electron density along the centerline for optically thin case. 


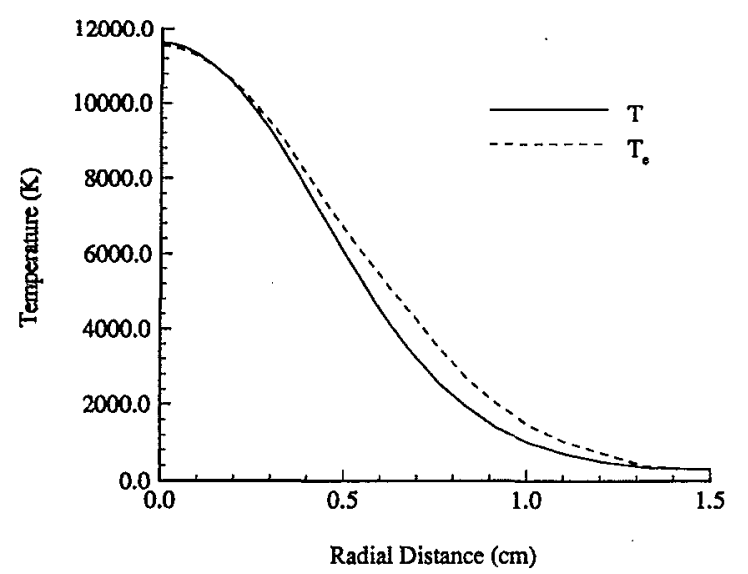

FIG. 9. Radial profiles of heavy particle and electron temperatures at $z=2$ $\mathrm{cm}$ for optically thick case.

For $z \geqq 3 \mathrm{~cm}$, the ions and excited state are no longer in equilibrium with the ground state, but the excited state continues to be in PLTE with the ions at the electron temperature. This too is consistent with the results of a previous study. ${ }^{13}$ In contrast, the optically thin results of Fig. 8 show large departures from ionization equilibrium with both the ground and excited states. These departures occur both upstream, where the electron density is high, and downstream to an increasing degree. Thus although the optically thin results show some interesting under- and overpopulation behavior, the closer agreement of the optically thick results with experimental observations for similar plasma temperatures and densities ${ }^{13}$ strongly indicates that the plasma is in fact optically thick to resonance radiation, and that selfabsorption of resonance radiation is indeed an important effect.

Radial profiles of heavy particle and electron temperatures at $z=2 \mathrm{~cm}$ are shown in Figs. 9 and 10 for both cases. In both cases, small departures from thermal equilibrium result from electron heating due to collisional recombination

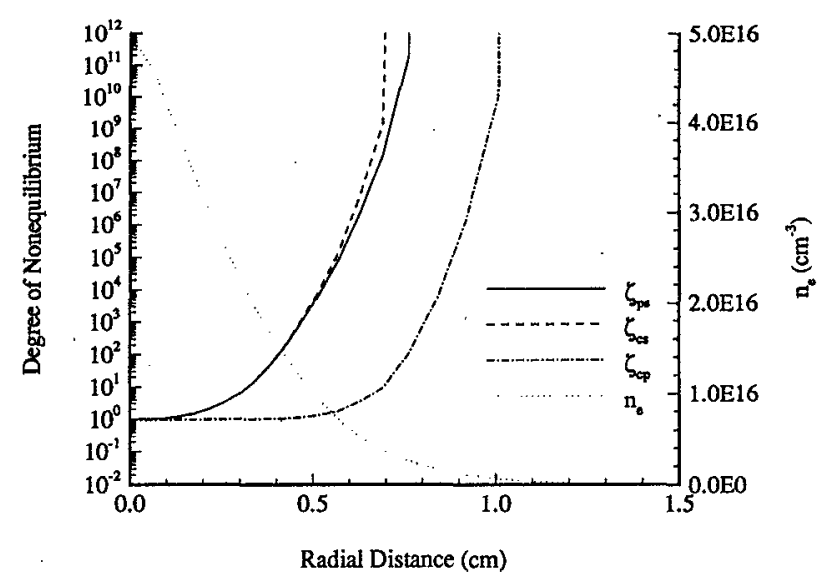

FIG. 11. Radial profiles of degree of nonequilibrium and electron density at $z=2 \mathrm{~cm}$ for optically thick case.

processes. In the optically thin case shown in Fig. 10, however, $T_{e}<T$ near the symmetry axis resulted from the resonance radiation escape as explained above. This effect also reduces $T_{e}$ in the fringes, thereby bringing $T_{e}$ down closer to $T$ than it is in the optically thick case (Fig. 9).

Figures 11 and 12 show radial profiles of degrees of nonequilibrium and $n_{e}$ at the same axial location. As expected, overpopulation of electrons and excited species occurs at the fringes of the jet, due to an insufficient separation between the chemical (collisional) and fluid dynamical time scales, as discussed below. In the optically thick case (Fig. 11), these overpopulations are further enhanced by absorption of resonance radiation. In the optically thin case (Fig. 12), the calculation showed underpopulation of excited and ionized species in the core part of the jet, also as expected. The optically thick case, Fig. 11, also shows that PLTE exists in much of the jet core. However, significant departures from PLTE are predicted around $n_{e}=10^{15} \mathrm{~cm}^{-3}$ in contrast to Fig. 7, where PLTE is predicted at the same electron density. This

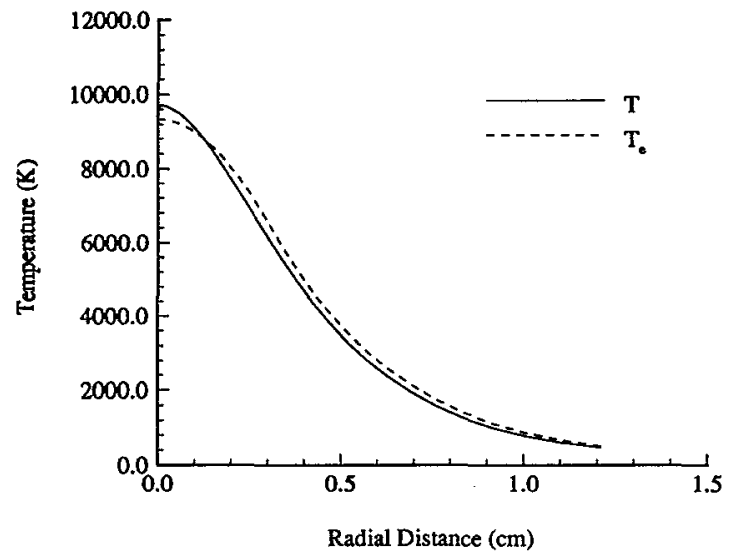

FIG. 10. Radial profiles of heavy particle and electron temperatures at $z=2$ $\mathrm{cm}$ for optically thin case.

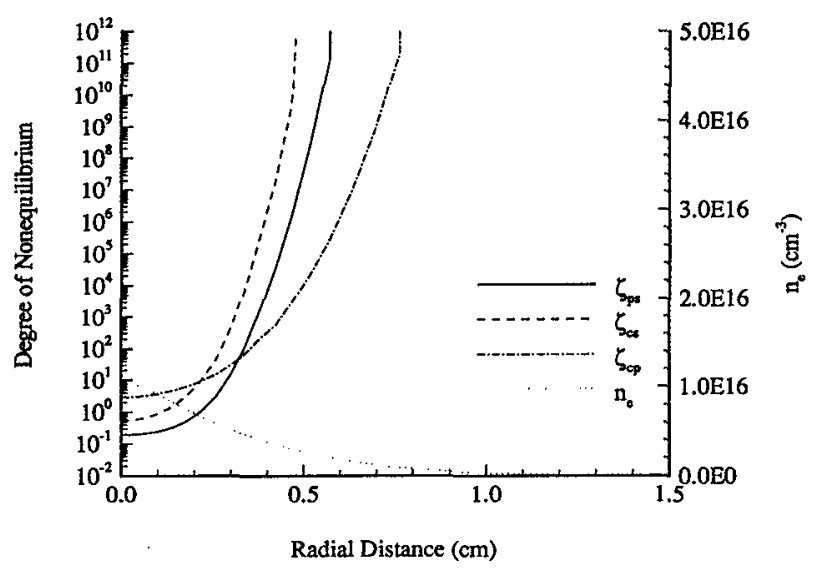

FIG. 12. Radial profiles of degree of nonequilibrium and electron density at $z=2 \mathrm{~cm}$ for optically thin case. 
illustrates the important point that $n_{e}$ itself has no absolute significance; what is really relevant are the time scales.

It is apparent that the relative size of the various time scales plays a decisive role in all of these nonequilibrium effects, since the thermodynamic state of the plasma is determined by competing physical effects with different time scales. The correspondence between PLTE and relative time scales can be analyzed in a semiquantitative way by plotting the time scales for the various processes as functions of position. This analysis will be limited to the optically thick case.

The appropriate reference frame for time scale considerations is a local Lagrangian frame moving with the plasma. (The plasma velocity relative to the laboratory frame is irrelevant, since the equations are invariant to Galilean transformations.) Fluid dynamical processes produce deviations from chemical equilibrium in a Lagrangian fluid element by changing the species densities and temperature. The temperature changes usually dominate, since equilibrium constants depend very strongly on temperature. The time scale for species density changes due to fluid expansion or contraction is $\tau_{e}=|\nabla \cdot \mathbf{u}|^{-1}$, while that due to diffusion of species $k$ is $\tau_{d}=\rho_{k}\left|\nabla \cdot \mathbf{J}_{k}\right|^{-1}$. The local fluid dynamical time scales corresponding to temperature changes are the times over which these changes significantly alter the equilibrium constant $K$ of the reaction under consideration; i.e., $\tau=K|D K / D t|^{-1}=|(d \ln K / d T) D T / D t|^{-1}$, where $D / D t$ $=\partial / \partial t+\mathbf{u} \cdot \nabla$ is the convective derivative. We shall restrict attention to the effects of fluid expansion $(e)$ and heat conduction $(q)$, for which $D T / D t \sim T \nabla \cdot \mathbf{u}$ and $\left(\rho c_{v}\right)^{-1} \nabla \cdot \mathbf{q}$, respectively.

These various fluid dynamical time scales are then to be compared with the local chemical time scale for the reaction $c$ under consideration; i.e., $\tau_{c}=\rho_{k}\left|\dot{\rho}_{k 1}^{c}\right|^{-1}$, where species $k$ is the participating species of smallest concentration, and $\dot{\rho}_{k !}^{c}$ is the unidirectional (i.e., forward or backward, not net) chemical rate of change of $\rho_{k}$. In the present context, the species of smallest concentration is the excited state $p$, and we shall therefore evaluate both $\tau_{c}$ and $\tau_{d}$ for $k=p$. The fluid dynamical and chemical time scales for deexcitation $(c=x)$ are therefore given by

$$
\begin{aligned}
& \frac{1}{\tau_{e x}}=\left|\frac{d \ln K_{p s}}{d T_{e}}\right| T_{e}|\nabla \cdot \mathbf{u}|, \\
& \frac{1}{\tau_{q x}}=\left|\frac{d \ln K_{p s}}{d T_{e}}\right| \frac{1}{\rho c_{v}}|\nabla \cdot \mathbf{q}|, \\
& \frac{1}{\tau_{x}}=\frac{1}{\rho_{p}}\left|\left(\dot{\rho}_{p 1}^{c}\right)_{x}\right|,
\end{aligned}
$$

while those for recombination to the excited state $(c=r)$ are

$$
\begin{aligned}
& \frac{1}{\tau_{e r}}=\left|\frac{d \ln K_{c p}}{d T_{e}}\right| T_{e}|\nabla \cdot \mathbf{u}|, \\
& \frac{1}{\tau_{q r}}=\left|\frac{d \ln K_{c p}}{d T_{e}}\right| \frac{1}{\rho c_{v}}|\nabla \cdot \mathbf{q}|, \\
& \frac{1}{\tau_{r}}=\frac{1}{\rho_{p}}\left|\left(\dot{\rho}_{p 1}^{c}\right)_{r}\right|,
\end{aligned}
$$

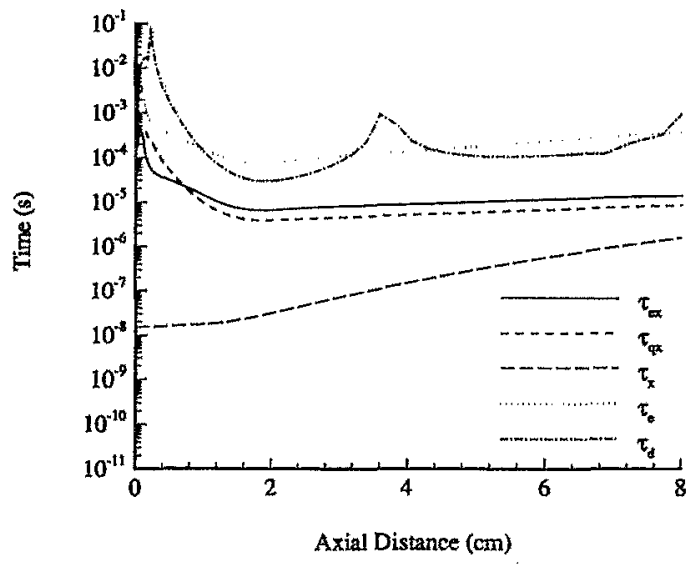

FIG. 13. Time scales for deexcitation along the centerline.

$$
\frac{1}{\tau_{r r}}=\frac{1}{\rho_{p}}\left|\left(\dot{\rho}_{p 1}^{c}\right)_{r r}\right|
$$

where subscript $r r$ represents optically thin radiative recombination. The time scales $\tau_{e}$ and $\tau_{d}$ are of course common to both deexcitation and recombination.

Figures 13 and 14 show the various time scales along the centerline for deexcitation and recombination, respectively. The sharp peaks in the expansion time scales near $z=0$ are due to the fact that there is a transition from compression to expansion there, so that $\boldsymbol{\nabla} \cdot \mathbf{u}$ changes sign and passes through zero. The peaks in $\tau_{d}$ are similarly due to sign changes in $\nabla \cdot J_{p}$. The time scales $\tau_{e}$ and $\tau_{d}$ are seen to be longer than the time scales associated with temperature changes, confirming that the temperature changes are indeed dominant. Comparison of Figs. 7 and 13 shows that departures from excitation equilibrium occur when the chemical deexcitation time scale $\tau_{x}$ approaches within two orders of magnitude of the time scales $\tau_{e x}$ and $\tau_{q x}$, which occurs for $z \gtrsim 3 \mathrm{~cm}$. (It might appear surprising that such a large separation of time scales is required for equilibrium, but the pre-

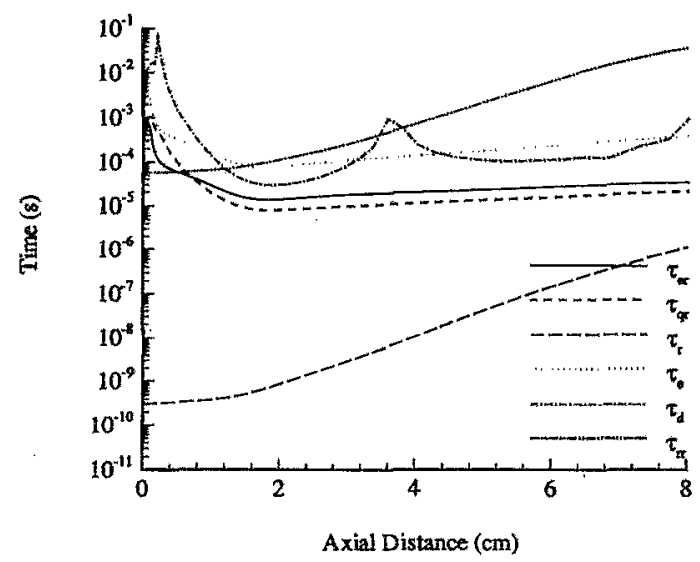

FIG. 14. Time scales for recombination to the excited state along the centerline. 


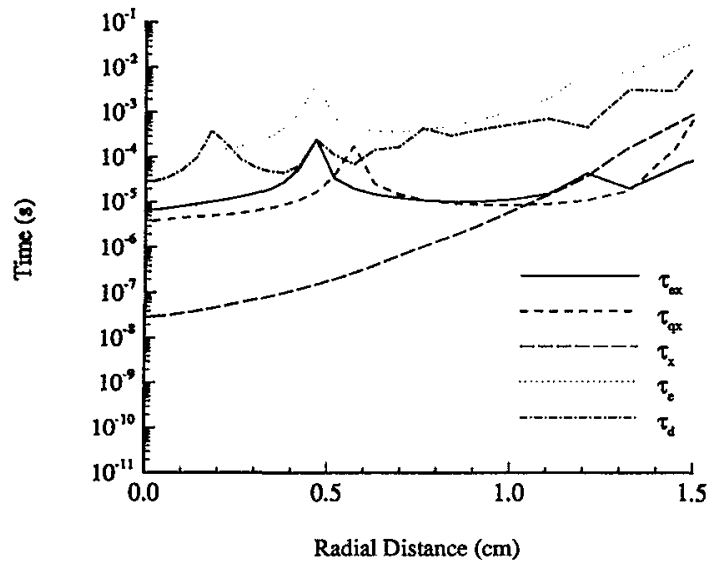

FIG. 15. Radial profiles of time scales for deexcitation at $z=2 \mathrm{~cm}$.

cise magnitude of this separation should not be taken too seriously; it depends on the definitions of the time scales, which are inherently imprecise quantities.) Comparison of Figs. 7 and 14 shows that recombination behaves similarly: when the chemical recombination time scale $\tau_{r}$ approaches within two orders of magnitude of $\tau_{e r}$ and $\tau_{q r}$, which occurs at $z \simeq 6 \mathrm{~cm}$, departures from PLTE are observed. Notice that the radiative recombination time scale $\tau_{r r}$ is considerably slower than $\tau_{e r}$ and $\tau_{q r}$ except in the immediate vicinity of $z=0$. Radiative recombination therefore does not contribute significantly to the departures from equilibrium.

Similar correlations are observed in the radial direction. Figures 15 and 16 are radial plots of the various time scales at $z=2 \mathrm{~cm}$, which show that $\tau_{e}, \tau_{d}$, and $\tau_{r r}$ are again generally slower than the other time scales and therefore do not play a significant role in the departures from equilibrium. (The peaks in these plots are again associated with sign changes in $\boldsymbol{\nabla} \cdot \mathbf{u}, \boldsymbol{\nabla} \cdot \mathbf{q}$, and $\boldsymbol{\nabla} \cdot \mathbf{J}_{p}$. ) Once again, when the chemical time scales $\tau_{x}$ and $\tau_{r}$ approach within two orders of magnitude of $\tau_{e x}, \tau_{q x}, \tau_{e r}$, and $\tau_{q r}$ (which occurs at $r \geqslant 0.2$ $\mathrm{cm}$ for deexcitation, and at $r \geq 0.5 \mathrm{~cm}$ for recombination),

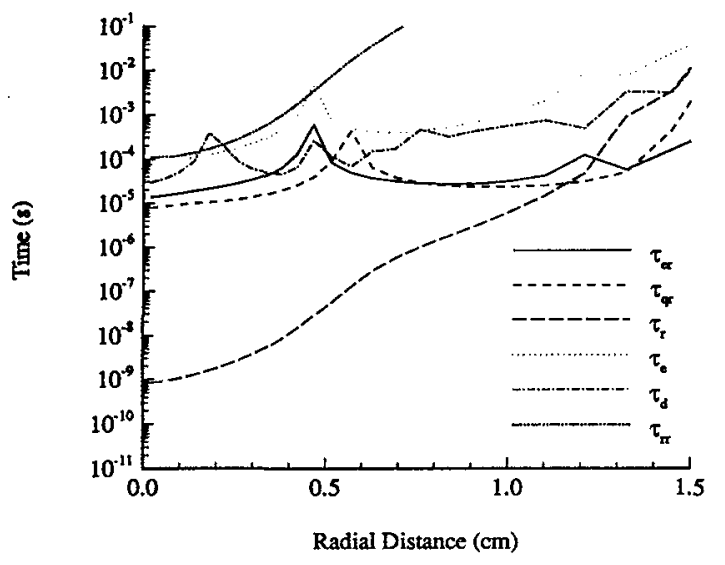

FIG. 16. Radial profiles of time scales for recombination to the excited state at $z=2 \mathrm{~cm}$.

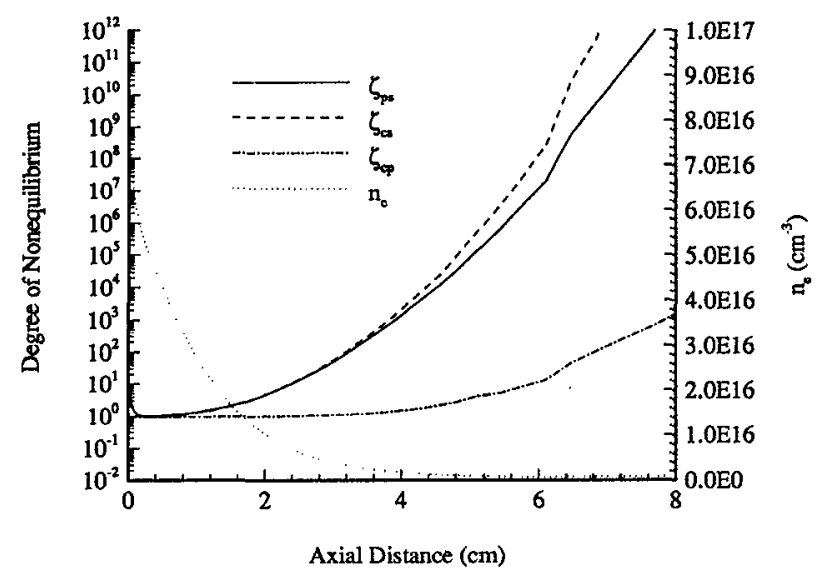

FIG. 17. Degree of nonequilibrium and electron density along the centerline in the low power case.

Fig. 11 shows departures from excitation equilibrium or PLTE in the same locations. These comparisons clearly show that the thermodynamic state of the plasma relative to equilibrium is determined by the relative time scales of competing physical and chemical processes, just as one would intuitively expect. These time scales are in turn determined by a variety of other variables and their gradients, including temperature, plasma velocity, and concentrations. Thus electron number density alone cannot and does not characterize or determine the LTE or PLTE status of a plasma.

For purposes of comparison, we also performed a similar optically thick simulation with lower values of the torch power and argon mass flow rate, using the same reduced grid as in the preceding optically thin calculation. The parameter values for this simulation were $P=1.2 \mathrm{~kW}$ and $W=0.148$ $\mathrm{g} / \mathrm{s}$, resulting in $v_{0}=106.14 \mathrm{~m} / \mathrm{s}$ and $T_{0}=12506 \mathrm{~K}$ with $n=3$ and $m=5$. Since $v_{0}$ is much slower than in the previous high speed case, one might have expected the plasma to be closer to LTE or PLTE. However, plasma velocity alone does not define a relevant time scale, and significant departures from ionization and excitation equilibrium still occur. Figure 17 shows electron density and degree of nonequilibrium in this case, while the relevant time scales are shown in Figs. 18 and 19. The correspondence between PLTE and time scale ratios is seen to be generally similar to that in the high-speed case.

\section{CONCLUDING REMARKS}

Upper excited states are not included in the present study. Extrapolating from the behavior of the first excited state, however, we anticipate that PLTE of upper excited states may well occur in some regions where departures from PLTE of the lower excited states occur. It would be necessary, however, to include more excited states in the model to confirm this conjecture and to predict the behavior of spectroscopic measurements as well as the relationship between the excited state kinetics and ionization reactions.

Previous studies ${ }^{13,14}$ have often discussed PLTE in terms of plasma electron density. While this is not unreasonable in a quiescent plasma, it can be very misleading in a flowing 


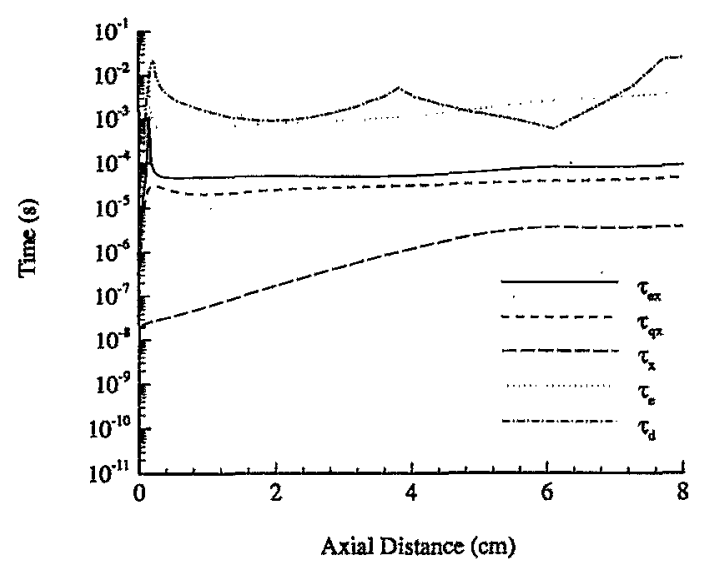

FIG. 18. Time scales for deexcitation along the centerline in the low power case.

plasma. The essential condition required for LTE or PLTE is that the relevant chemical time scales be much shorter than the competing fluid dynamical time scales. The electron density itself has no absolute significance (nor do fluid parameters such as flow velocity, as our low-speed simulation illustrates).

We have shown that complete simulations of the plasma flow and excited state kinetics permit a detailed analysis of LTE and PLTE effects, and that these effects may be interpreted in terms of the local time scale ratios existing in different parts of the plasma. In the present simulations, an insufficient separation between chemical and fluid dynamical time scales drives the plasma away from thermal and ionization equilibrium in spite of the fact that complete LTE has been assumed at the inflow boundary. Recent experimental observations of a free burning $\operatorname{arc}^{29}$ using direct temperature measurement techniques ${ }^{30}$ indicate that significant departures

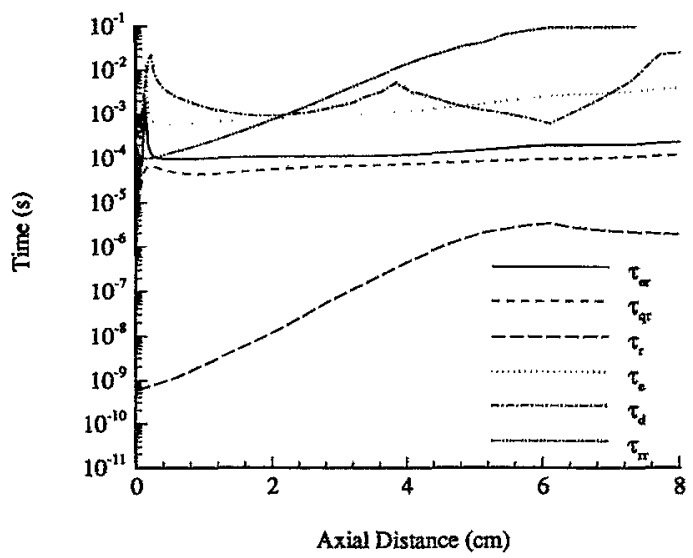

FIG. 19. Time scales for recombination to the excited state along the centerline in the low power case. from thermal equilibrium occur in the core of the arc, thereby providing a further source of nonequilibrium effects in plasma plumes generated by electric arcs. The nonequilibrium behavior of plasma jets utilizing experimentally determined nonequilibrium boundary conditions is currently under investigation, and will be reported in due course.

\section{ACKNOWLEDGMENTS}

This work was performed under the auspices of the U.S. Department of Energy under DOE Field Office, Idaho, Contract No. DE-AC07-76ID01570, supported by the U.S. Department of Energy, Office of Energy Research, Office of Basic Energy Sciences, Division of Engineering and Geosciences.

'M. Mitchner and C. H. Kruger, Partially lonized Gases (Wiley, New York, 1973).

${ }^{2}$ G. W. Sutton and A. Sherman, Engineering Magnetohydrodynamics (McGraw-Hill, New York, 1965).

${ }^{3} \mathrm{C}$. H. Chang and E. Pfender, Plasma Chem. Plasma Process. 10, 473 (1990).

${ }^{4}$ C. H. Chang and E. Pfender, Plasma Chem. Plasma Process. 10, 493 (1990).

${ }^{5}$ C. H. Kruger, Phys. Fluids 13, 1737 (1970).

${ }^{6} \mathrm{~J}$. Mostaghimi and M. I. Boulos, in Proceedings of the 9th International Symposium of Plasma Chemistry (ISPC-9) (International Union of Pure and Applied Chemistry, Pugnochiuso, Italy, 1989), Vol. 1, p. 91

${ }^{7}$ C. H. Chang and E. Pfender, IEEE Trans. Plasma Sci, PS-18, 958 (1990).

${ }^{8} \mathrm{H}$. Petschek and S. Byron, Ann. Phys. 1, 270 (1957).

${ }^{9}$ M. I. Hoffert and H. Lien, Phys. Fluids 10, 1769 (1970).

${ }^{10} \mathrm{C}$. G. Braun and J, A. Kunc, Phys. Fluids 30, 499 (1987).

"T. E. Repetti, J. R. Fincke, and W. A. Neuman, in Heat Transfer in Thermal Plasma Processing, edited by K. Etemadi and J. Mostaghimi (American Society of Mechanical Engineers, New York, 1991). HTD, Vol. 161, pp. $167-175$

${ }^{12}$ R. S. Lee. AIAA J. 2, 637 (1964).

${ }^{13}$ T. G. Owano, C. H. Kruger, and R. A. Beddini, AIAA J. 31, 75 (1993).

${ }^{14}$ M. H. Gordon and C. H. Kruger, Phys. Fluids B 5, 1014 (1993).

${ }^{15}$ K. Y. Cho and T. L. Eddy, J. Quant. Spectrosc. Radiat. Transfer 41, 287 (1989).

${ }^{16}$ J. D. Ramshaw and C. H. Chang, Plasma Chem. Plasma Process. 12, 299 (1992).

${ }^{17}$ C. H. Chang and J. D. Ramshaw, Plasma Chem. Plasma Process. 13, 189 (1993).

${ }^{18}$ J. D. Ramshaw and C. H. Chang, Plasma Chem. Plasma Process. 11, 395 (1991).

${ }^{19}$ J, D. Ramshaw and C. H. Chang, Plasma Chem. Plasma Process. 13, 489 (1993).

${ }^{20}$ T. Holstein, Phys. Rev. 72, 1212 (1947).

${ }^{21}$ T. Holstein, Phys, Rev. 83, 1159 (1951).

22 J. D. Ramshaw, J. Non-Equilib. Thermodyn. 15, 295 (1990).

${ }^{23}$ J. D. Ramshaw, J. Non-Equilib. Thermodyn. 18, 121 (1993).

${ }^{24} \mathrm{R}$. Aris, Vectors, Tensors, and the Basic Equations of Fluid Mechanics (Prentice-Hall, Englewood Cliffs, 1962).

${ }^{25}$ B. E. Launder, A. Morse, W. Rodi, and D. B. Spalding, Prediction of Free Shear Flow-A Comparison of the Performance of Six Turbulence Models (National Aeronautics and Space Administration, Washington, DC, 1973), NASA SP-321. Vol. 1, p. 361

${ }^{26} \mathrm{M}$. A. Leschziner and W. Rodi. AIAA J. 22, 1742 (1984)

${ }^{27}$ J. R. Fincke, C. H. Chang, W. D. Swank, and D. C. Haggard, Int. J. Heat Mass Transfer 37, 1673 (1994).

${ }^{28}$ J. D. Ramshaw and C. H. Chang, "Iteration scheme for implicit calcula" tions of kinetic and equilibrium chemical reactions in fluid dynamics." $\mathrm{J}$. Comput. Phys. (to be published).

${ }^{29}$ S. C. Snyder, G. D. Lassahn, and L. D. Reynolds, Phys. Rev. E 48, 4124 (1993).

${ }^{30}$ S. C. Snyder, L. D. Reynolds, G. D. Lassahn, J. R. Fincke, C. B. Shaw, and R. Kearney, Phys. Rev. E 47, 1996 (1993). 